\title{
Feuille de route pour la physique des deux infinis
}

Sandrine Emery (sandrine.emery@cea.fr) ${ }^{(1)}$ et Sotiris Loucatos ${ }^{(1,2}$

(1) Institut de Recherche sur les lois Fondamentales de I'Univers (IRFU), CEA-Saclay, 91191 Gif-sur-Yvette Cedex

(2) Laboratoire AstroParticule et Cosmologie (APC), Université Paris Diderot-Paris 7.

En avril 2012, durant les journées

de prospective des instituts

IN2P3 du CNRS et IRFU du CEA,

les chercheurs et ingénieurs

ont exposé et débattu

de leurs recommandations

aux directions des instituts

afin d'établir une feuille de

route en physique nucléaire,

en physique des particules et

des astroparticules, en France

pour les dix années à venir.

Cette réunion consacrait

une année de préparation

approfondie et de rédaction

de documents par différents

groupes de travail.

Concernant la physique

des particules, les débats

et les synthèses servent

de base à la suite au niveau

européen : la stratégie

européenne en cours d'élabo-

ration par le Conseil du CERN.
Deux instituts français sont dédiés à la recherche fondamentale en physique subatomique [1] : l'Institut National de Physique Nucléaire et de Physique des Particules (IN2P3) [2] du CNRS et l'Institut de Recherche sur les lois Fondamentales de l'Univers (IRFU) [3] de la Direction des Sciences de la Matière (DSM) du CEA. L'IN2P3 et l'IRFU collaborent étroitement sur leurs thématiques de recherche en grande partie communes $^{(a)}$ et couvrant la physique nucléaire, la physique des particules et des astroparticules. Cette coordination est d'autant plus cruciale que ces recherches nécessitent souvent de très grands et coûteux instruments et que les projets s'étalent sur de longues échelles de temps, de la conception à l'analyse des données : parfois plus de vingt ans, comme pour le Large Hadron Collider (LHC) [4], à l'origine de la récente découverte d'une nouvelle particule qui semble bien être le boson de Higgs ${ }^{(b)}$ mythique tant recherché, signature de la théorie fondamentale qui lie la nature du vide à la génération des masses des particules élémentaires [5]. Ces projets nécessitent également collaborations et coordinations internationales, européennes et au-delà, le tout dans un contexte en constante évolution, marqué par des avancées de la recherche ou des impacts sur les projets liés à la conjoncture économique et politique. Il s'agit donc dès à présent de préparer les projets qui démarreront entre 2020 et 2030, tout en gardant suffisamment de souplesse dans la stratégie.

Une réunion de prospective IN2P3-IRFU [6] a eu lieu à Giens, dans le Var, du 2 au 5 avril 2012, pour proposer aux directions des deux instituts une feuille de route pour 2013-2022, avec des extrapolations à plus long terme. Les précédentes et premières journées de ce type avaient eu lieu en 2004, renforçant les liens déjà engagés lors de rencontres entre 1'IN2P3 et la DSM. Aussi, au cours d'une année de travail approfondi, vingt groupes de travail thématiques ont préparé chacun un document d'orientation soumis aux commentaires de notre communauté jusqu'aux présentations orales et la discussion de Giens. Les groupes de travail couvrent l'essentiel des grands domaines de recherche en physique nucléaire (structure du nucléon, structure et dynamique nucléaire, astrophysique nucléaire, physique et chimie du cycle électronucléaire), en physique des particules (étude du Modèle Standard et mesures de précision, violation de la symétrie matière-antimatière, neutrinos, recherche de physique au-delà du Modèle Standard), à la frontière des deux spécialités (plasma de quarks-gluons), de même que l'étude de l'Univers comme laboratoire (astroparticules, tests de la gravitation, recherche d'ondes gravitationnelles, cosmologie, matière noire et énergie noire). D'autres groupes de travail se sont consacrés aux recherches à la frontière avec d'autres disciplines (interfaces avec les sciences de la vie et de la Terre), aux domaines transverses indispensables au bon fonctionnement des recherches (théorie, accélérateurs, instrumentation et détection, calcul), aux relations recherche-enseignement et à l'organisation et au financement de la recherche.

Les conclusions des groupes de travail liés à la physique des particules ont été soumises comme contributions à la stratégie européenne préparée par le CERN [7], abordée plus loin. Les documents complets d'orientation et les synthèses sont disponibles en [6]. Les premières conclusions ont été données à la fin du colloque de Giens par les directeurs des deux instituts, Jacques Martino (IN2P3) et Philippe Chomaz (IRFU). En avril 2012, Jacques Martino a adressé une lettre à ce sujet au personnel de l'IN2P3 [8]. On pourra par ailleurs consulter, pour davantage d'informations sur les projets, les sites de l'IN2P3 [2] et de l'IRFU [3]. Concernant les astroparticules et la cosmologie, le site du laboratoire APC [9] offre une présentation pédagogique. 

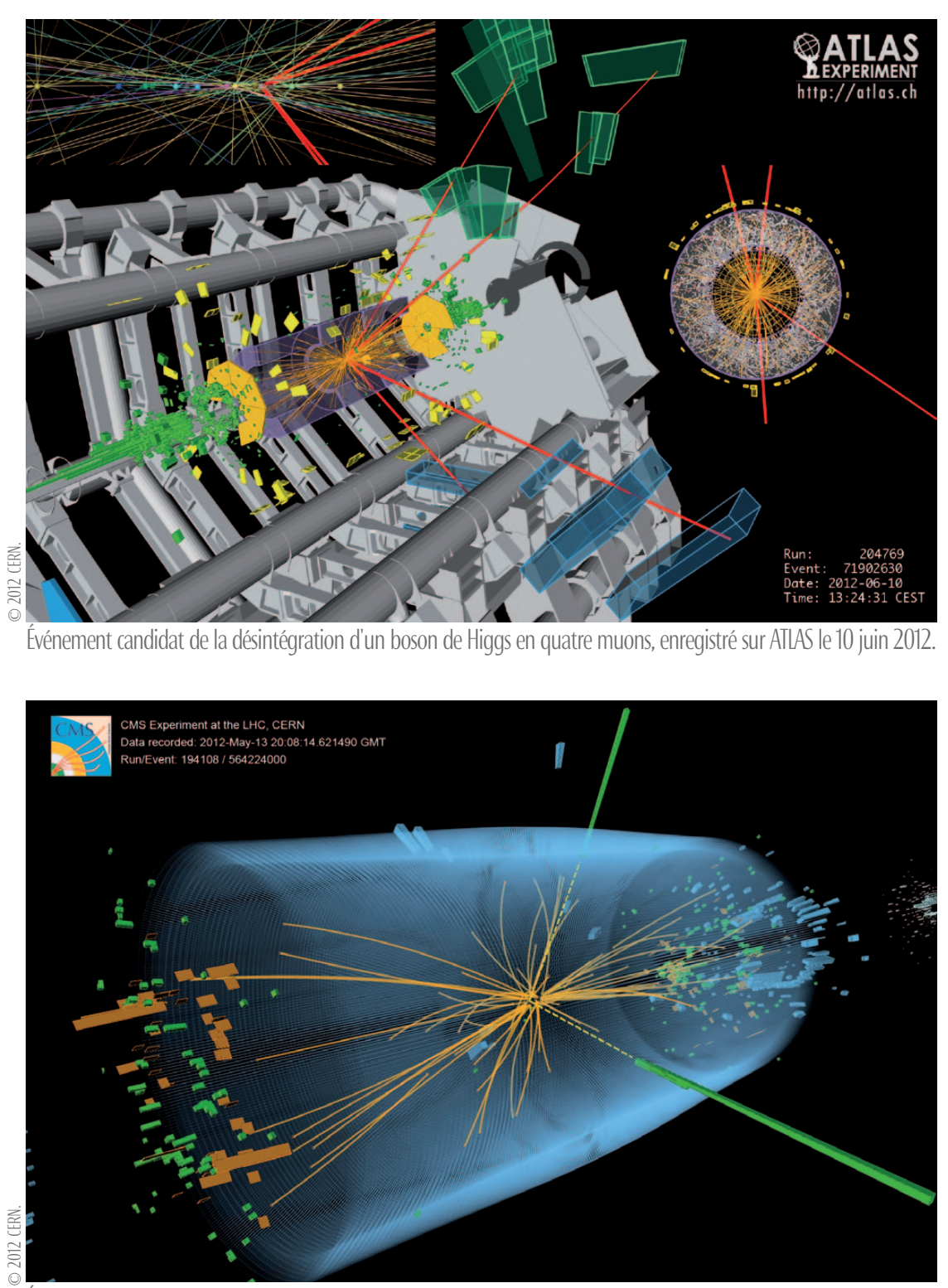

Événement candidat de la désintégration d'un boson de Higgs en deux photons, enregistré sur CMS le 4 juillet 2012.

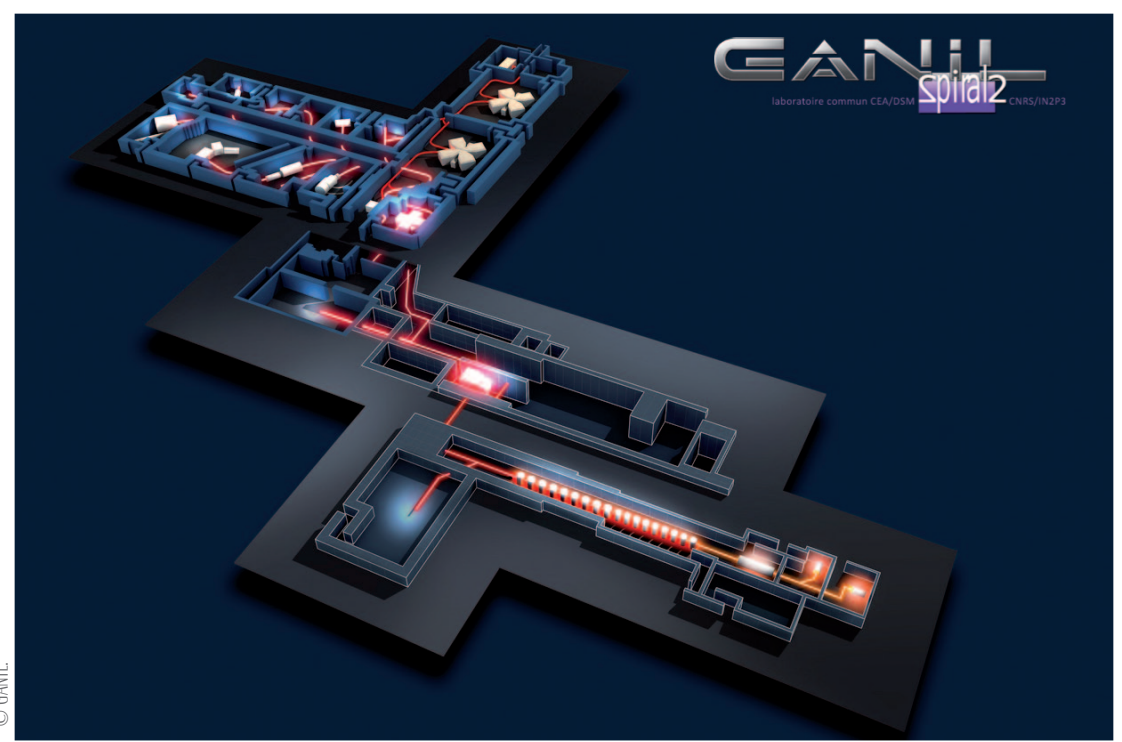

Vue d'artiste de l'accélérateur SPIRAL2, au GANIL à Caen.

\section{\>}

Les premières recommandations des journées de prospective soulignent une volonté marquée de pousser plus loin les études engagées dans chacun des domaines de recherche abordés. Cependant pour certains domaines, les débats sont encore très animés quant aux moyens et projets qui pourraient être retenus et aux échelles de temps associées.

En physique nucléaire, les objectifs sont très clairs autour du projet phare GANIL/ SPIRAL2 [10]. Le GANIL (Grand Accélérateur National d'Ions Lourds), installé à Caen pour la recherche française et européenne, est un $\mathrm{GIE}^{(c)}$ CNRS-CEA piloté par l'IN2P3 et la DSM. SPIRAL2 (Système de Production d'Ions RAdioactifs en Ligne de $2^{\text {e }}$ génération) est une nouvelle installation de production et d'accélération de noyaux exotiques unique au monde, qui démarrera prochainement. Elle permettra d'étendre l'étude des propriétés fondamentales des noyaux et d'apporter de nouvelles contraintes à la description de l'interaction entre nucléons, qui est à la base de la structure microscopique des noyaux. Au sein de cette installation, une nouvelle source de neutrons, appelée NFS (Neutrons for Science) sera consacrée à l'étude des réactions nucléaires induites par neutrons, ce qui présente un intérêt aussi bien fondamental qu'appliqué dans un large spectre de thématiques, allant de la physique atomique à la physique des matériaux et à la radiobiologie. La stratégie européenne pour la physique nucléaire a été débattue et définie en 2010 au sein du comité NuPECC [11].

Les débats sont bien engagés en physique des particules, notamment sur les futurs instruments possibles pour l'étude précise du nouveau boson apparenté au boson de Higgs et pour la recherche d'une nouvelle physique au-delà du Modèle Standard, au-delà du programme déjà prévu au LHC au moins jusqu'à 2020. D'autres accélérateurs seront probablement nécessaires pour répondre aux nouvelles questions. Une physique au-delà du Modèle Standard pourrait être découverte soit par des déviations révélées à l'aide de mesures de grande précision dans des collisions $\mathrm{e}^{+} \mathrm{e}^{-}$ (voire $\mu^{+} \mu^{-}$), soit par la découverte de particules de grande masse sur des machines hadroniques d'énergie beaucoup plus haute. Les prospectives IN2P3-IRFU ont eu lieu quelques mois avant l'annonce de la découverte du nouveau boson, même si les résultats du LHC laissaient déjà entrevoir 
son existence. Diverses options ont été considérées, suivant la confirmation ou non de ce boson. Les futurs résultats du LHC qui, selon le programme prévu jusqu'en 2020, devraient s'appuyer sur un quasidoublement de l'énergie actuelle des collisions et sur une augmentation sensible de l'intensité des faisceaux, pourraient aussi changer les éléments de base des décisions. L'étude en cours d'un projet d'accélérateur linéaire électron-positron $\left(\mathrm{e}^{+} \mathrm{e}^{-}\right)$[12] complémentaire du LHC, pour lequel le Japon est actuellement en première ligne, suscite des débats sur l'engagement possible de l'Europe, engagement qu'il faut considérer dans le contexte d'autres projets, au CERN par exemple.

En plein essor depuis une douzaine d'années, la physique des neutrinos ouvre une fenêtre d'étude vers une nouvelle physique, et fait également l'objet de vives discussions. La confirmation expérimentale [13] du changement de saveur des neutrinos muoniques en neutrinos électroniques est apparue quasiment au moment de la tenue des journées de prospective. De grandes questions encore ouvertes portent sur l'asymétrie matière-antimatière dans le secteur des leptons et l'ordre relatif des masses des neutrinos.

Dans le domaine des astroparticules, l'intérêt des grands projets en préparation (exposés par exemple en [9]) a été confirmé (LSST et Euclid ${ }^{(\mathrm{d})}$ pour l'énergie noire, CTA ${ }^{(\mathrm{e})}$ pour les gammas de hautes énergies, Advanced Virgo et LISA ${ }^{(\mathrm{f})}$ pour les ondes gravitationnelles...). Parmi les projets de détection directe de matière noire, sujet fondamental pour la compréhension de l'Univers, un choix parmi les technologies en compétition doit être fait (détecteurs à cristaux de germanium, au xénon liquide...). La coordination de la physique des neutrinos et des astroparticules en Europe se fait par l'ApPEC [14].

L'abandon du projet SuperB ${ }^{(\mathrm{g})}[15]$, très prometteur pour l'étude de l'asymétrie matière-antimatière, a été annoncé en novembre 2012 en Italie pour des raisons de coût bien plus élevé que prévu. Cela reporte donc les recherches associées à ce domaine principalement au Japon [13] et sur l'expérience dédiée $\mathrm{LHCb}$ du LHC.

Les réflexions sur le long terme sont donc difficiles, car des découvertes importantes et des événements économiques et politiques peuvent changer le cours des orientations. De plus, si les réflexions sont basées essentiellement sur les objectifs de physique, elles doivent aussi inclure une coordination au niveau mondial avec les autres projets, et des considérations stratégiques liées à l'avenir de la recherche dans les différentes régions du monde et à celui des grands laboratoires comme le CERN.

Suite des réflexions au niveau européen et au-delà. En physique des particules, les discussions se sont prolongées avec la préparation de la stratégie européenne menée par le "European Strategy Group” (ESG), pour le conseil du CERN [16]. Un symposium ouvert à Cracovie en septembre 2012 [17], a recueilli les contributions et les avis des physiciens européens. Les premières conclusions de ces réflexions sont exposées en [18].

Concernant les recherches sur le boson de Higgs et sur une éventuelle nouvelle physique, les questions qui se posent sont : avons-nous besoin d'autres machines pour étudier les propriétés du boson ? Si c'est le cas, après prise en compte des facteurs tels que la maturité technologique, le coût et le lieu, quelle sera la machine optimale : un collisionneur $\mathrm{e}^{+} \mathrm{e}^{-}$linéaire ou circulaire, un collisionneur de photons ou un collisionneur de muons?

Quelles sont les options pour une future machine en Europe après le LHC ? Sera-ce une machine pour traiter la physique à l'échelle de $10 \mathrm{TeV}$ ? Est-ce que les données du LHC à l'énergie nominale fourniront une justification suffisante pour cela ? Quand sera le bon moment pour prendre une décision, et quel type de R \& D doit être fait pour se préparer à une telle décision dans le futur?

La communauté européenne du neutrino prépare un programme de recherche avec des faisceaux de ligne de base longue pour mesurer les paramètres de mélange des saveurs de neutrinos. La communauté des astroparticules propose que le détecteur lointain nécessaire pour ce programme soit aussi compatible avec un programme de recherche de désintégration du proton et de détection des neutrinos cosmiques. En parallèle, plusieurs projets sont en cours pour l'étude des neutrinos dits "stériles". Mais doit-on raisonner seulement dans le cadre européen ou l'effort mondial doit-il être coordonné ?

Un document détaillé [19] sert de base aux réflexions de l'ESG qui se réunit à Erice fin janvier 2013, pour soumettre au conseil du CERN une proposition de remise à jour de la stratégie européenne. Le Conseil prendra sa décision à Bruxelles en mai 2013.

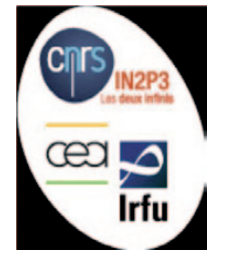

(a) La différence entre les deux instituts dans l'organisation des grandes thématiques se situe au niveau de l'astrophysique et de la physique théorique. L'IRFU comprend un service d'astrophysique à part entière tandis qu'au CNRS, l'astrophysique non liée à la physique nucléaire ou des particules, est traitée par l'Institut National des Sciences de l'Univers (INSU). Au CEA, l'Institut de Physique Théorique (IPhT) traite l'ensemble de la physique théorique, incluant les thématiques de l'IRFU et de l'IN2P3, alors que des physiciens théoriciens sont directement associés à l'IN2P3.

(b) Plus exactement, le boson de Robert Brout, François Englert et Peter Higgs (BEH).

(c) GIE : Groupement d'Intérêt Économique

(d) LSST et Euclide : télescopes respectivement au sol et satellisé pour l'étude des structures à grande échelle de l'Univers.

(e) CTA : Cerenkov Telescope Array, détecteur de gerbes atmosphériques.

(f) Avanced Virgo et LISA : interféromètres, respectivement au sol et dans l'espace.

(g) SuperB : machine de production de mésons contenant le quark lourd «b " ou "beauté ».

\section{Références}

1• Introduction à la physique subatomique dans la revue Élémentaire : http://elementaire.web.lal.in2p3.fr

2• www.in2p3.fr

3• http://irfu.cea.fr/index.php

4 www.lhc-france.fr

5• Reflets de la physique n 31 (octobre 2011), pp. 17-19.

6• http://journeesprospective.in2p3-irfu.fr

7• Stratégie européenne : http://europeanstrategy group.web.cern.ch/europeanstrategygroup

8• http://listserv.in2p3.fr/cgi-bin/ $w a ? A 2=$ ind $1204 \& L=e-$ lettre- $\mid \& T=0 \& P=58$

9• www.apc.univ-paris7.fr/APC_CS

10• www.ganil-spiral2.eu/spiral2

11- "Perspectives of Nuclear Physics in Europe": www.nupecc.org/Irp2010/Documents/Irp2010 final_hires.pdf

12• www.linearcollider.org/

13- Reflets de la physique n`29 (mai 2012), p. 17.

14• www.aspera-eu.org

15• http://physicsworld.com/cws/article/news/2012/ nov/28/italy-cancels-euro-1bn-superb-collider

16• http://council.web.cern.ch/council/fr/Bienvenue.html

17- http://espp2012.ifj.edu.pl ; présentations sur le site : http://indico.cern.ch/conferenceDisplay. py?confld $=182232$

18• http://europeanstrategygroup.web.cern.ch/european strategygroup/ESPG/espg_2012_009_final.pdf ; Courrier du CERN du 6 novembre 2012 http://cerncourier.com/cws/article/cern/51170

19• https://indico.cern.ch/materialDisplay.py?contribld $=2 \&$ materialld $=1 \&$ confld $=217861$ 